\title{
Instability of isolated boundary-layer streaks to spatially- developing travelling waves
}

DOI:

10.1016/j.euromechflu.2019.04.011

\section{Document Version}

Accepted author manuscript

Link to publication record in Manchester Research Explorer

\section{Citation for published version (APA):}

Hewitt, R., \& Duck, P. (2019). Instability of isolated boundary-layer streaks to spatially-developing travelling waves. European Journal of Mechanics B. Fluids, 76, 413-421. https://doi.org/10.1016/j.euromechflu.2019.04.011

\section{Published in:}

European Journal of Mechanics B. Fluids

\section{Citing this paper}

Please note that where the full-text provided on Manchester Research Explorer is the Author Accepted Manuscript or Proof version this may differ from the final Published version. If citing, it is advised that you check and use the publisher's definitive version.

\section{General rights}

Copyright and moral rights for the publications made accessible in the Research Explorer are retained by the authors and/or other copyright owners and it is a condition of accessing publications that users recognise and abide by the legal requirements associated with these rights.

\section{Takedown policy}

If you believe that this document breaches copyright please refer to the University of Manchester's Takedown Procedures [http://man.ac.uk/04Y6Bo] or contact uml.scholarlycommunications@manchester.ac.uk providing relevant details, so we can investigate your claim.

\section{OPEN ACCESS}




\section{Accepted Manuscript}

Instability of isolated boundary-layer streaks to spatially-developing travelling waves

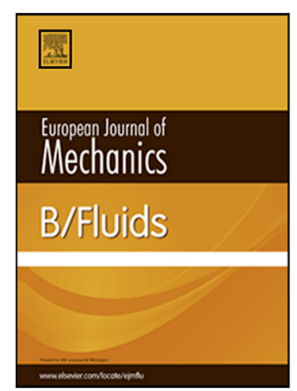

PII: S0997-7546(18)30757-X

DOI: https://doi.org/10.1016/j.euromechflu.2019.04.011

Reference: EJMFLU 3470

To appear in: European Journal of Mechanics / B Fluids

Received date : 17 December 2018

Revised date: 11 April 2019

Accepted date : 16 April 2019

Please cite this article as: R.E. Hewitt and P.W. Duck, Instability of isolated boundary-layer streaks to spatially-developing travelling waves, European Journal of Mechanics / B Fluids (2019), https://doi.org/10.1016/j.euromechflu.2019.04.011

This is a PDF file of an unedited manuscript that has been accepted for publication. As a service to our customers we are providing this early version of the manuscript. The manuscript will undergo copyediting, typesetting, and review of the resulting proof before it is published in its final form. Please note that during the production process errors may be discovered which could affect the content, and all legal disclaimers that apply to the journal pertain. 


\title{
Instability of isolated boundary-layer streaks to spatially-developing travelling waves
}

\author{
R.E. Hewitt*, P.W. Duck \\ School of Mathematics, University of Manchester, UK.
}

\begin{abstract}
The spatial stability properties of an isolated low-speed streak e $\mathrm{h}_{\mathrm{h}} \mathrm{Aded}$ in a Blasius boundary layer are determined; the streak is generated by steady localised injec. ' $n$, $w^{h}{ }^{h}$ ' $t$ the disturbance is generated by a linear harmonic localised injection. Isolated streaks driven by hort-scale spanwise forcing have comparable growth rates of both sinuous and varicose instabli : as. T ese features have been discussed previously via DNS methods, but the novel aspect here is a tre ' 'ment via a rationally-parabolised version of the Navier-Stokes equations in the high Reynolds nur- ${ }^{-1} \ldots$.... The parabolic formulation allows for a more efficient and Reynolds number independent compula : $n$ of fully three-dimensional non-parallel streaks their stability. We compute the non-parallel $d \epsilon u_{r}$ - ant of a perturbation by downstream marching from a time-harmonic disturbance generator (in $\operatorname{ta}_{1}{ }^{-} \mathrm{s}$ with the streamwise streak development), before comparing these results with bi-global eige.' $v$ ' '1e , alculations. The stability properties are well captured by a weakly non-parallel eigenvalue formu. tion of the boundary-region equations, provided that one is not in the vicinity of the disturbance ener a.or. Further downstream, or at higher excitation frequencies we directly recover the long-wave limil of a two-dimensional Rayleigh stability problem. Even further downstream (or at even high $\ldots$ i itation frequencies, which is mathematically equivalent) we must return to a (two-dimensional) ayleigh formulation as the streamwise wavelength of the disturbance becomes comparable to the 'oun $\mathrm{rv}$ iayer thickness. For streaks that are comparable with those obtained experimentally our $\mathrm{s}^{r} \mathrm{atia}^{\prime}$ growth rates and eigenmode shapes compare favourably with the experimentally-determined values. For ne range of streaks considered, we demonstrate the sinuous mode retains the higher gror $h$ rate in the viscous stability problem. The experimentally-observed change over to a dominant var cose. nde nearer the disturbance site is shown to be true only for frequencies that provoke an invi „.. response.
\end{abstract}

Keywords: boundary layer, $s$ ㄱ ‘lity, transition, streaks

\section{Introduction and forme. 'on}

Localized surface $r$ ' ighnes, is known to have a substantial impact on the route of lamninar-turbulent transition in bor ary-ıay er flows, and there are a number of experimental/numerical studies that examine the stabilit: of flow over a three-dimensional roughness element. These surface-mounted roughness elements are typ allv ylindrical [1, 2], hemispherical [3, 4], conical [5], rectangular cuboid [6], mesh screens [7, . ' nmoth) bumps [8], and placed on otherwise flat plate in a uniform free stream. As a consequence ' $f$ ihe presence of the roughness element, in the 'wake' there develops streamwise-aligned vortices with as ociated streamwise streaks. Whilst streaks tend to be more prominent for sharp-sided

*richard.hewitt@manchester.ac.uk 
roughness elements, the generic features of the streaks are consistent across a range of disturbance generators, not limited to just surface roughness, but also including (for example) surfac inju tion over the same length scales. This is to be expected at least in the far downstream limit, bec . . a the recent work of [9] has demonstrated that a downstream-decaying streak in a Blasius boundary aye can be described (in this linearised regime) in terms of algebraic, steady, localised, self-similar, ri-glu 11 eigenmodes.

For carefully chosen distributions of three-dimensional surface roughness it is pos hle to stabilise the two-dimensional Tollmien-Schlichting mechanism $[10,11]$ and for similar $s$ ale . face injection (in the presence of a free stream pressure gradient) it is possible to prevent separativ. ' $\left.{ }^{2} 2\right]$. However in all cases, as the height of the surface roughness (or strength of the surface injectic ., is includsed, the streak that is induced downstream of the disturbance region ultimately becomes uns tble, lec ling to transition.

The problem of determining the stability of streaks has been tacklod … iange of approaches, for example, experimentally [7], numerically via global stability ana yses ..1 ' ${ }^{\prime}$ DNS [1, 13], as well as local parallel theories [14], global optimal perturbations [15] and in umpr ssible flow through bi-global eigenvalue computations and three-dimensional PSE analyses [6]. In some cases the analysis points to sinuous modes dominating the response, whilst in others va icose mo des are suggested to be the most relevant. Furthermore, even in sub-critical cases where var 'nse a. ' $d$ snuous modes are both stable, optimal perturbations can still achieve large growth [2]. In [1] botı 'inuous and varicose modes have been located as global eigenmodes for flow past (circular) cy."ndrical roughness elements, with the aspect ratio of the cylinder playing a major role in the selection of the lominant mode.

An approach that has not been considered in as much it . Il (and which we pursue herein) is one based on the assumption of an asymptotically large Re. - vlds umber. This methodology has a substantial simplifying advantage of formally reducing the gov $r n_{.}$g equations of both the base flow and perturbation to being parabolic systems. To pursu ' in ? roach we consider incompressible flow over a semi-infinite flat plate, which in the absence of any a 'ditional forcing merely results in the classical twodimensional Blasius solution. However, $\cdots \cdots$ focus here lies in the spatially developing instability of a single streak structure embedded in this $\mathrm{T}^{\text {/ lasius } ~} \mathrm{ow}$, as induced by a spatially localised boundary injection. This localised injection could ecuarı, he $r .1$ isolated roughness element, but one that is elongated in the streamwise direction to match t ie rf quired bounadry-layer scales. The resulting elongated streaks are $O\left(R e^{-1 / 2}\right) \times O\left(R e^{-1 / 2}\right)$ in the ( ${ }^{\prime} \cdot{ }^{\prime}$ nsic dess) cross-sectional plane in the high Reynolds number $(\operatorname{Re} \gg 1)$ limit. As such, any high Reyno: number reduction must retain both transverse and spanwise diffusion, therefore only diffusi in a d pressure gradients in the downstream direction are neglected in this approach.

Investigations that focus on ae ( $e \gg 1)$ stability of a single streak structure are less common than those that assume periodicity in the $s_{\mathrm{F}}$ ' $n$ wise direction. These isolated longitudinal streaks are more challenging in that the flow cor pon nts in the cross-sectional plane decay towards the free stream only algebraically as shown in [ $\mathrm{l}$,' dlthr dgh the corresponding vorticity and streamwise components still decay exponentially). This $\mu$ ebraic sehaviour is not found in two-dimensional or classical three-dimensional boundary layers ( $\mathrm{s}$ ? for ex mple $[17,18]$ ) and computational formulations that do not capture the far field correctly can $b_{c}{ }^{-t_{n}}$ sly affected by domain truncation. Having obtained a spatially-developing steady streak $\mathrm{r} \iota \mathrm{se}$ flo , any linear stability problem can be approached via the same formulation, although any loc. ${ }^{1}$ eigen alue analysis is then best formulated as a bi-global problem.

As noted ir the context of roughness elements above, isolated low-speed streaks are interesting in that, for sufficien 'v mall spanwise scales, the sinuous and varicose instabilities have been shown to have comparable gre vth rates. This has been demonstrated both experimentally by Asai et al. [7] and in the corresponding DNS results of Brandt [13]. Motivated by the experimental work reported in [7], the subsequent work of Brandt [13] used a fully three-dimensional, unsteady, spectral formulation of the Navier-Stokes system. The experimental arrangement of [7] employed a mesh screen attached to a flat 
plate in a uniform flow, to generate a steady streak. This mesh disturbance was mod lled in [13] as a volumetric forcing of the streamwise momentum equation with parameters chosen o ( $a_{\mathrm{f}}$. roximately) reproduce the steady streak data. To assess the stability of this streak the line : $-d$ Navier-Stokes equations were time marched from an impulse response. The later stage nonlinear sea' down of unstable sinuous/varicose perturbations induced by time-harmonic boundary forcing was alsu 'nnsidered in [13]. The results of Brandt effectively reproduce the experimental behaviour found hy $r$ si et al. [7] but at substantial computational cost. Our goal here is to show that the same featur $s \mathrm{ca}: \mathrm{s}$ nroduced by a selfconsistent high Reynolds number asymptotic theory based around the 'boun ' ' $y$-region equations'. We focus particularly on $[7,13]$ because a mesh screen 'roughness' has been nuwn nu. to lead to reverse flow in the immediate wake, and so seems the most likely candidate for an ipplicat on of the high-Reynolds number theory.

This general type of 'boundary-region' formulation has been $\mathrm{p}$ 2vic' $r$, employed in (for example) discussions of isolated streaks $[16,19,20]$, spanwise-periodic struns/vo ices [21, 22], their transient growth $[23,24,25]$ and their self-sustained interaction with travel. - o waves [26].

In terms of a dimensional coordinate system $\left(x^{*}, y^{*}, z^{*}\right)$ ali ${ }^{\circ}$ ' ${ }^{\prime}$ wit the leading edge $\left(x^{*}=0\right)$, we non-dimensionalise with an arbitrary lengthscale $L^{*}$ in the $u_{c}$ 'al $w_{u_{y}}$ via

$$
x^{*}=L^{*} x, \quad\left(y^{*}, z^{*}\right)=* n-\frac{1}{2}(2 x)^{\frac{1}{2}}(\eta, \zeta),
$$

where $R e=U_{\infty}^{*} L^{*} / \nu^{*} \gg 1$ is a Reynolds number sed । ๆ a (uniform) freestream speed $U_{\infty}^{*}$ and kinematic viscosity $v^{*}$. In (1) the factor of $(2 x)^{1 / 2}$ is a cu venient method of capturing the downstream thickening/broadening of the viscous layer bu ne tions we consider have no self-similarity constraint. To allow a later formulation of the linear sta' $i$ ility problem for time-harmonic perturbations, we retain the unsteady terms with a correspon ${ }^{\mathrm{N} i n} \sigma$ choice for the natural timescale of $t^{*}=t L^{*} / U_{\infty}^{*}$ (this in itself precludes a self similar response).

The dimensional velocity field $\left(u^{*}, v^{*}, v^{*}\right) \mathrm{lo} \cdot \mathrm{xr}$.nded in the usual manner

$$
u^{*}=U_{\infty}^{*} U+\cdots \quad\left(1, w^{*}\right)=U_{\infty}^{*} R e^{-\frac{1}{2}}(2 x)^{-\frac{1}{2}}(V, W)+\cdots,
$$

where, in general, $U, V, W$ are $\mathrm{f}$ ' nctu 's of $x, \zeta, \eta, t$. The corresponding pressure field is

$$
p^{*}=\rho U_{\infty}^{*}\left(\operatorname{Re}^{-1 / 2} p(x, t)+\operatorname{Re}^{-1} P(x, \zeta, \eta, t)\right)+\cdots,
$$

in the absence of any free ,tream pr ssure gradient, with $\rho^{*}$ the constant density. Here $p$ is a displacement induced correction to $\mathrm{t}^{\prime}$ a $\mathrm{p}^{r}: \mathrm{ssu}^{\prime}:$ field that does not affect the leading-order system, whilst $P$ must be retained in the leading-orde. $m$,mentum equations in the high Reynolds number limit.

The leading-order $s$ /stem $\mathrm{t}$ " large Re provides a primitive variable formulation of the "boundary-region equations', however ' $n$ wha follows we pursue an alternative formulation that arises from a cross differentiation of the $.10 \mathrm{mentum}$ equations to usefully eliminate the pressure correction $P$, following previous authors, includ ng [9]. In a further substitution of

$$
(V, W)=(\eta U-\Phi, \zeta U-\Psi),
$$

we recover the 'imensionless unsteady boundary-region equations in the form given in [16], but now 
including the $x, t$ derivatives:

$$
\begin{gathered}
2 x U_{x}+2 U=\Phi_{\eta}+\Psi_{\zeta}, \\
\Theta=\Psi_{\eta}-\Phi_{\zeta}, \\
\nabla^{2} U=2 x U U_{x}-\Phi U_{\eta}-\Psi U_{\zeta}+2 x U_{t}, \\
\nabla^{2} \Theta=2 x\left(U_{\eta} \Psi_{x}-U_{\zeta} \Phi_{x}+U \Theta_{x}-\Theta U_{x}\right) \\
+2\left(\zeta U U_{\eta}-\eta U U_{\zeta}\right)-\Phi \Theta_{\eta}-\Psi \Theta_{\zeta}-2 U \Theta+2 x
\end{gathered}
$$

where

$$
\nabla^{2} \equiv \frac{\partial^{2}}{\partial \eta^{2}}+\frac{\partial^{2}}{\partial \zeta^{2}}
$$

As a streak generation mechanism, we allow for $O\left(R e^{-1 / 2}\right)$ injec. ' $n$ thr' on the $\eta=0$ boundary, leading to the boundary conditions

$$
\begin{gathered}
U=\Psi=0, \quad \Phi=\Phi_{i n j}(x, \zeta, t, \quad \text { O1s } \quad l=0, \\
U \rightarrow 1, \quad \Psi \rightarrow 0, \quad \text { aс } n \rightarrow \text { 。 },
\end{gathered}
$$

where $\Phi_{i n j}$ defines an injection distribution on the plate surfac. This localised injection is used to drive a steady streak base state for a subsequent linear stabı ${ }^{\prime}+y$; laı $y$ sis.

The far-field behaviour of (4) is analysed in detai ' $n$ the vork of Hewitt et al. [16]. Of particular note here is that

$$
(\Phi, \Psi) \sim \frac{A(x, t)}{\zeta^{2}+\eta^{2}}\left(\eta, \zeta, \quad \text { for } \quad \zeta^{2}+\eta^{2} \gg 1\right.
$$

away from the boundary, leading to alg Jraic $~ '$ cay into the free stream of the cross-sectional $(V, W)$ velocities, although the vorticity compon nt defi ed by $\Theta$ in (4b) still decays exponentially. For the solutions presented herein, we enforce $t^{\prime}$. 1s algeu. $1 \mathrm{c}$ behaviour explicitly to improve accuracy of solutions computed on a truncated domain, $w^{\prime}$ ich urn out to be vitally important for numerical accuracy.

From the definition (2), this bound ary inju ${ }^{\circ}$ on $\Phi_{i n j}$ corresponds to a weak dimensional injection velocity evaluated at the wall of

$$
\left.v^{*}{ }^{*}{ }^{*}=0\right)=-U_{\infty}^{*} R e^{-\frac{1}{2}}(2 x)^{-\frac{1}{2}} \Phi_{i n j}(x, \zeta, t) .
$$

Our focus is on injections $\left(\Phi_{i n j}-0\right)$ that are localised in space with $\left|\Phi_{i n j}\right| \rightarrow 0$ exponentially away from the injection region.

\section{Non-parallel in ection Iriven steady streaks}

Before we addre the _...uility properties, we first consider a class of steady non-parallel developing base states; for this we xtend the recent approach of Hewitt et al. [9]. The downstream evolution of the steady flow car he det rmined by decomposing the solution into a two-dimensional Blasius base flow plus a (sho nanwise scale) finite-amplitude correction:

$$
\begin{gathered}
U=U_{B}(\eta)+\tilde{U}(x, \eta, \zeta), \\
\Phi=\Phi_{B}(\eta)+\tilde{\Phi}(x, \eta, \zeta), \\
\Psi=\zeta \Psi_{B}(\eta)+\tilde{\Psi}(x, \eta, \zeta), \\
\Theta=\zeta \Theta_{B}(\eta)+\tilde{\Theta}(x, \eta, \zeta) .
\end{gathered}
$$


In this approach, zero injection $\left(\Phi_{i n j} \equiv 0\right)$ leads to the (two dimensional) Blasius ate (denoted by the subscript- $B$ terms) being the only non-zero contribution. For $\Phi_{i n j} \neq 0$ this decr npu ${ }^{\circ}$ ion yields a nonlinear system of equations that must be marched numerically (parabolically) $\mathrm{d}$. . 'stream in $x$ from the leading edge for the flow quantities $(\tilde{U}, \tilde{\Phi}, \tilde{\Psi}, \tilde{\Theta})$.

We choose to induce a steady streak by a localised injection of the form

$$
\Phi_{i n j}(x, \zeta)=-\kappa F\left(x ; x_{0}\right) G(\zeta)
$$

where

$$
F\left(x ; x_{0}\right)=e^{-\gamma\left(x-x_{0}\right)^{2}} \text { and } G(\zeta)=e^{-C \zeta^{2}}\left(1-\alpha^{\prime} \zeta \zeta^{2}\right)
$$

in what follows we fix $\gamma=10$ as variations of $\gamma$ are equivalent tc a re $-\mathrm{a}$. ng of $x, x_{0}$. This is largely a Gaussian injection profile in both the downstream and spanwise cuvidina $e$, with a minor modification of the $\left(1-2 C \zeta^{2}\right)$ factor, which ensures a zero net mass flux into 4 . boundary layer. The magnitude of the injection is determined by $\kappa$, the downstream location $\mathrm{c}^{c}$ the inje tion is centred at $x=x_{0}$, whilst the (spanwise) lengthscale of the injection region decreases ' $\mathrm{c}$ r in - . .ing values of the constant $C$. This choice of injection is consistent with the recent paper of Hewitt ${ }^{+}$al. [9], but our focus is now instead on the stability properties of the resulting steady structurc.

The baseflow evolution is determined by discretisati of $(4)$ in the $(\zeta, \eta)$ plane, using a second-order central finite-difference scheme. The discretisation is v vf a uniform mesh in a transformed (computational) coordinate system, leading to a non-unifc. . mes in the $(\zeta, \eta)$ plane. The downstream evolution is determined by Newton iteration applied at $\mathrm{e}_{\mathrm{c}} \mathrm{h}$ i ownstream $x$ location, using a routine CrankNicolson method for the downstream derivativ. $\quad 2 .-{ }_{n}$ esults in a mesh of $N_{\zeta} \times N_{\eta}$ nodal points and a $\left(4 N_{\zeta} N_{\eta} \times 4 N_{\zeta} N_{\eta}\right)$ sparse matrix inversion for each 1 woton iteration, which is handled via a multifrontal

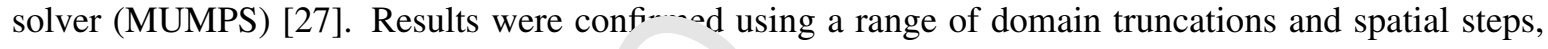
with typical values for moderate injecti n valut being $N_{\zeta}=N_{\eta}=401$ for $(\zeta, \eta) \in[0,20] \times[0,20]$, whilst a typical downstream spatial sten $w_{m} \cdot \delta x \cdot 0.005$.

In figure 1 we show contours of dor nstr am velocity $U$ in the cross sectional $(\zeta, \eta)$ plane for $\kappa=12$, $x_{0}=10$. The contours are shown bou, " ar $\mathrm{t}^{\prime} \mathrm{e}$ injection location $x=11$ (left column) and further downstream at $x=14$ (right column). ariations in the width of the injection region (via $C=0.1,0.05,0.025$ ) are shown from top to bottom i: the.. rure. The steady injection (9) was shown by Hewitt \& Duck [9] to be a good quantitative approx: ... ' ion of the steady streak experiments of [7] over a broad range of downstream positions. We theref re : sply (9) as a convenient computational proxy to such experimentallyobserved localised streaks. Ln a for an experimentally obtained base flow from [7] are also shown in figure 1(a) and we will $r$ turr to discuss our results in this experimental context in section 4 .

\section{Linear stability of a lo vlised-streak}

Our ultimate ge : is to un ermine the stability of such steady streak states to linearised unsteady (time harmonic) pert rbation, which result in downstream propagating, spatially developing waves. We tackle this problem vic a cor oination of parabolic marching of the linearised disturbance equations, together with a loca $\ldots$-nvalue analysis in the context of the boundary-region formulation (4).

For base flow such as those shown in figure 1, driven by injection profiles described above in (9), we consider the linear stability to perturbations of a set forcing frequency $\omega$ (a real constant) via a 

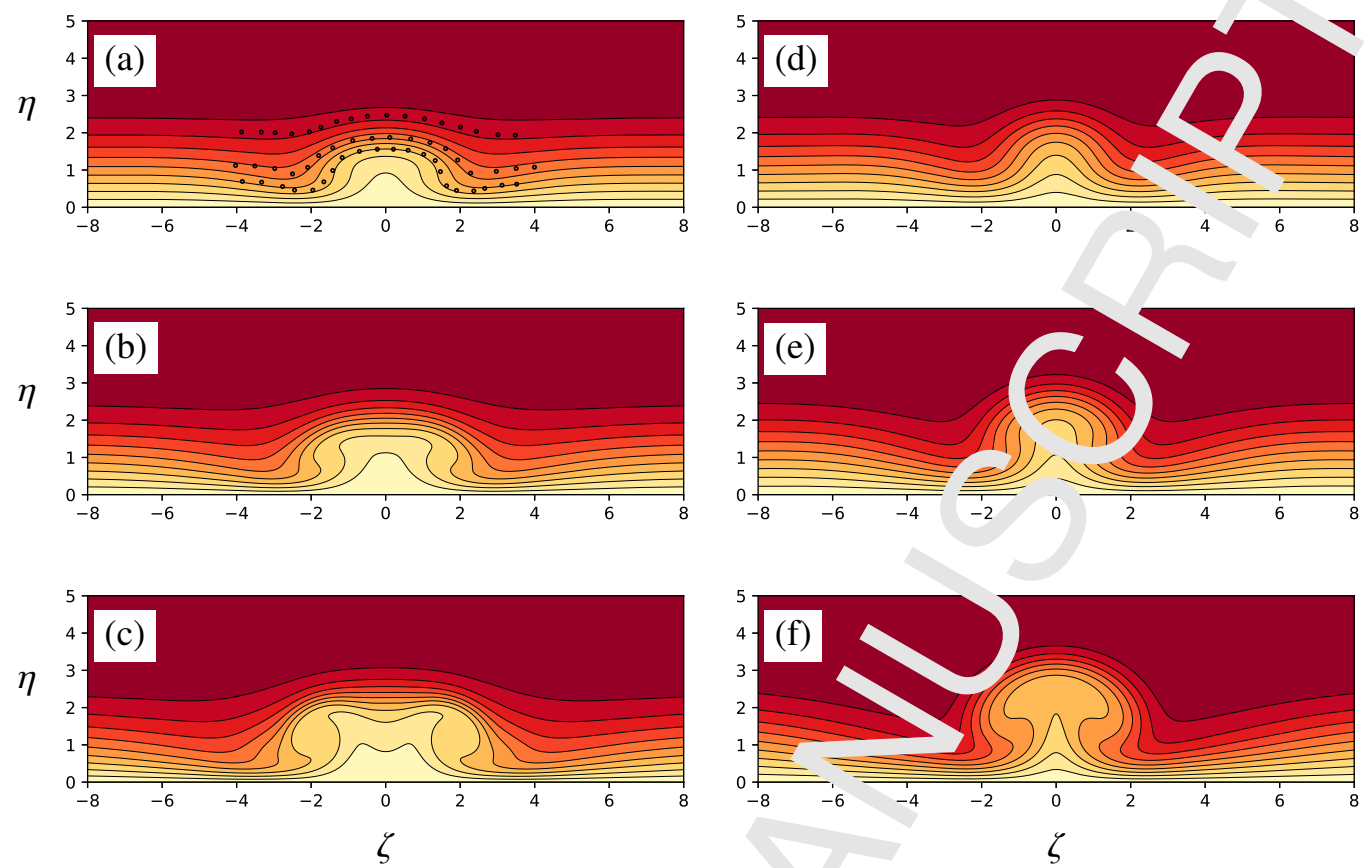

Figure 1: Examples of the steady developing localised streak 'seflor as measured by the downstream velocity $U$. The streak is induced by injection at $x_{0}=10$, with an injection parameter $t_{n}-12$. Two different downstream locations are shown, (a-c) at $x=11$ and (d-f) further downstream at $x=14$. The snanwise . idth of the injection slot increases from top to bottom, with (a,d) $C=0.1,(\mathrm{~b}, \mathrm{e}) C=0.05$ and $(\mathrm{c}, \mathrm{f}) C=0.025$. The 、 ntous ure shown at $U=0.1,0.2, \ldots, 0.9$. Data points in (a) are experimental results for $U=0.3,0.5,0.8$, as shown in figure 3(८, of [7].

decomposition into

$$
\begin{aligned}
& U=\left[U_{B}(\zeta)+\breve{J}(x . \eta, \zeta)\right]+\epsilon e^{-i \omega t} u(x, \eta, \zeta)+\text { c.c. }, \\
& \Phi=\left[\Phi_{\zeta}(\eta) . \tilde{\Phi}(., \eta, \zeta)\right]+\epsilon e^{-i \omega t} \phi(x, \eta, \zeta)+\text { c.c. }, \\
& \left.\Psi=' \zeta \chi_{\llcorner}(\eta)+\tilde{\Psi}(x, \eta, \zeta)\right]+\epsilon e^{-i \omega t} \psi(x, \eta, \zeta)+\text { c.c. }, \\
& \left.\Theta \quad \Theta_{B}(\eta)+\tilde{\Theta}(x, \eta, \zeta)\right]+\epsilon e^{-i \omega t} \theta(x, \eta, \zeta)+\text { c.c. . }
\end{aligned}
$$

For each component the squa bracketed terms determine the localised, nonlinear, non-parallel streak of (8), whilst the $O(\epsilon) \dagger, \mathrm{rms}$ are the time-harmonic spatially developing linear disturbance field. The base flows are such that $\tilde{r}_{T}$ s sy ımetric about the centreline $\zeta=0$, but the disturbance field can either be varicose (with $u \mathrm{~s}^{\mathrm{r}} \ldots$ metı $\ldots$ or sinuous (with $u$ antisymmetric).

To determine the $c$ swnstre m evolution of the $O(\epsilon)$ perturbation, we take two approaches. First we specify a form of inil. $1 \mathrm{f} \mathrm{n}^{*}$.ing, via a small scale harmonic injection superimposed onto (9), and numerically (parabol : ally) arch $(u, \phi, \psi, \theta)$ downstream in tandem with the spatially developing base flow. Secondly, we a 'dress t' e stability locally as a bi-global eigenvalue calculation; the results from the two approaches will be umpared. 

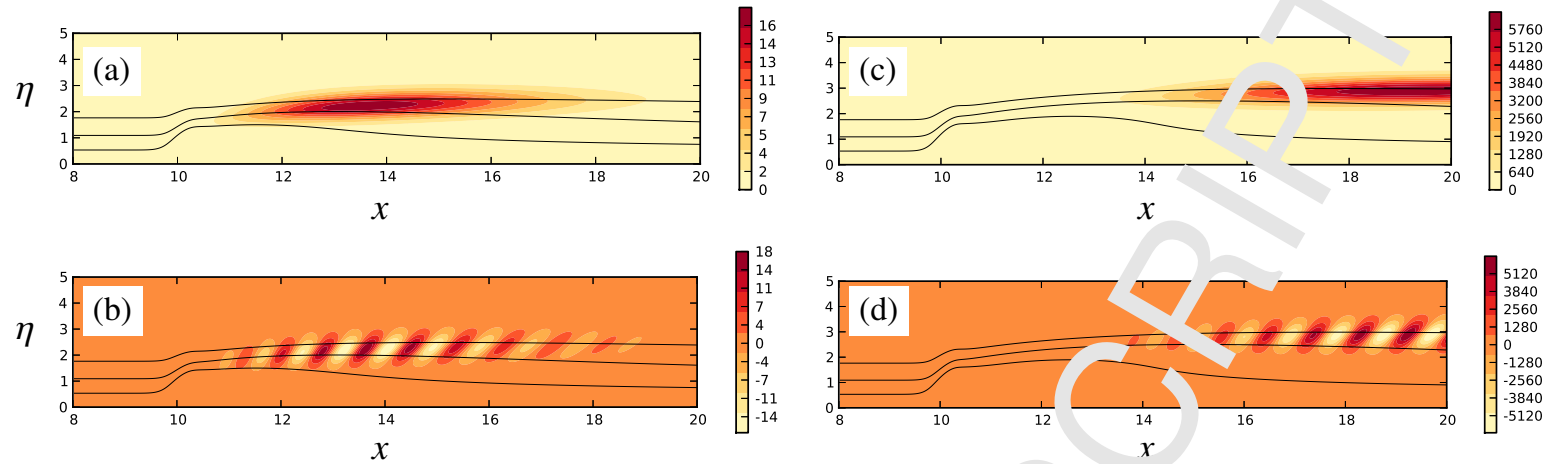

Figure 2: The centreline $\zeta=0$ evolution of a varicose linearised harmonic perth hr on of irequency $\omega=5$ for (a,b) $C=0.1$ and (c,d) $C=0.05$ for $\kappa=12$ and $x_{0}=10$. Contours show the absolu value ${ }^{\circ}$.ne perturbation $u(x, \eta, \zeta=0, \eta)$ in $(\mathrm{a}, \mathrm{c})$ and its real part in $(\mathrm{b}, \mathrm{d})$. In all cases the three contours shown as lines $1 \mathrm{1}$ " cate where the steady baseflow satisfies $U(\zeta=0, \eta)=0.25,0.5,0.75$. The perturbation is normalised such that the, sak value ,f $|u|$ is unity at $x=x_{0}$.

\subsection{Parabolic disturbance equations}

On writing $u(x, \eta, \zeta)=f(x, \eta, \zeta) / x$, the linearised $(\epsilon \ll 1) \mathrm{p}_{2}$ 'rbation equations arise from substitution of (10) into (4):

$$
\begin{aligned}
& 2 f_{x}=\ldots+\psi_{s} \\
& \theta=\psi_{\eta} \cdot \phi_{\zeta}, \\
& x^{-1}\left\{\nabla^{2} f+\left(\Phi_{B}+\tilde{\Phi}\right) f_{\eta}+\left(\zeta \Psi_{B}+\Psi,{ }_{\nu}{ }^{c}+2\left(U_{B}+\tilde{U}\right) f\right\}+\phi\left(U_{B}^{\prime}+\tilde{U}_{\eta}\right)+\psi \tilde{U}_{\zeta}\right. \\
& =2\left(U_{r} \cdot \tilde{I J}\right) f_{x}+2 f \tilde{U}_{x}-2 i \omega f, \\
& x^{-1}\left\{\nabla^{2} \theta+\left(\Phi_{B}+\tilde{\Phi}\right) \theta_{\eta}+\phi\left(\tilde{\Theta}_{.}+\zeta \Theta_{B}^{\prime}\right)+\psi\left(\Theta_{B}+\tilde{\Theta}_{\zeta}\right)+\theta_{\zeta}\left(\tilde{\Psi}+\Psi_{B}\right)+2\left(U_{B}+\tilde{U}\right) \theta\right\} \\
& \left.-2 x^{-2}\left(\zeta\left(U_{B}+j\right) f_{r}+\zeta_{J}, \tilde{U}_{\eta}+U_{B}^{\prime}\right)-\eta\left(U_{B}+\tilde{U}\right) f_{\zeta}-\eta f \tilde{U}_{\zeta}\right) \\
& =2\left(\left(U_{B}^{\prime}+\tilde{I}_{\eta)_{4}} .-\tilde{U}_{0} \phi_{x}+\left(U_{B}+\tilde{U}\right) \theta_{x}-x^{-1}\left(\zeta \Theta_{B}+\tilde{\Theta}\right) f_{x}\right)\right. \\
& \left.+2 \mu^{-1}\left(f_{\eta} \tilde{\Psi}_{x}-f_{\zeta} \tilde{\Phi}_{x}+f \tilde{\Theta}_{x}\right)-\theta \tilde{U}_{x}\right)-2 i \omega \theta .
\end{aligned}
$$

This system fully captures th $f_{\text {or }}$ tial development of a perturbation, provided that the disturbance exists on the same length scales a the underlying localised streak. Computational results require (parabolic) marching of (11) in additi $\mathrm{n}$ to $\mathrm{L}$. steady nonlinear streak flow, in $x$ from suitable initial conditions. This is achieved by applying the ame second-order Crank-Nicolson method as for the base flow. At each downstream position this $\mathrm{arc}^{\mathrm{n}}$ ng procedure provides the full disturbance field in the cross-sectional $(\eta, \zeta)$ plane, which i equivale $\mathrm{t}$ t to the $\left(y^{*}, z^{*}\right)$ plane via the rescaling (1).

To excite the varico a mode ve impose the same injection profile for the harmonic perturbation, as given in (9), via

$$
\phi(x, \zeta, \eta=0)=F\left(x ; x_{0}\right) G(\zeta),
$$

which corr ${ }_{u_{\mathrm{r}}}$-de to a linear amplitude modulation of the injection. To excite a sinuous mode we correspondin ${ }_{i} 1 \cdot$ impose

$$
\phi(x, \zeta, \eta=0)=F\left(x ; x_{0}\right) \zeta e^{-C \zeta^{2}} .
$$

Figure 2 shows the evolution of varicose perturbations, as measured by the downstream velocity perturbation $u$ evaluated at the centreline $\zeta=0$. Shown are the development of two varicose perturbations 


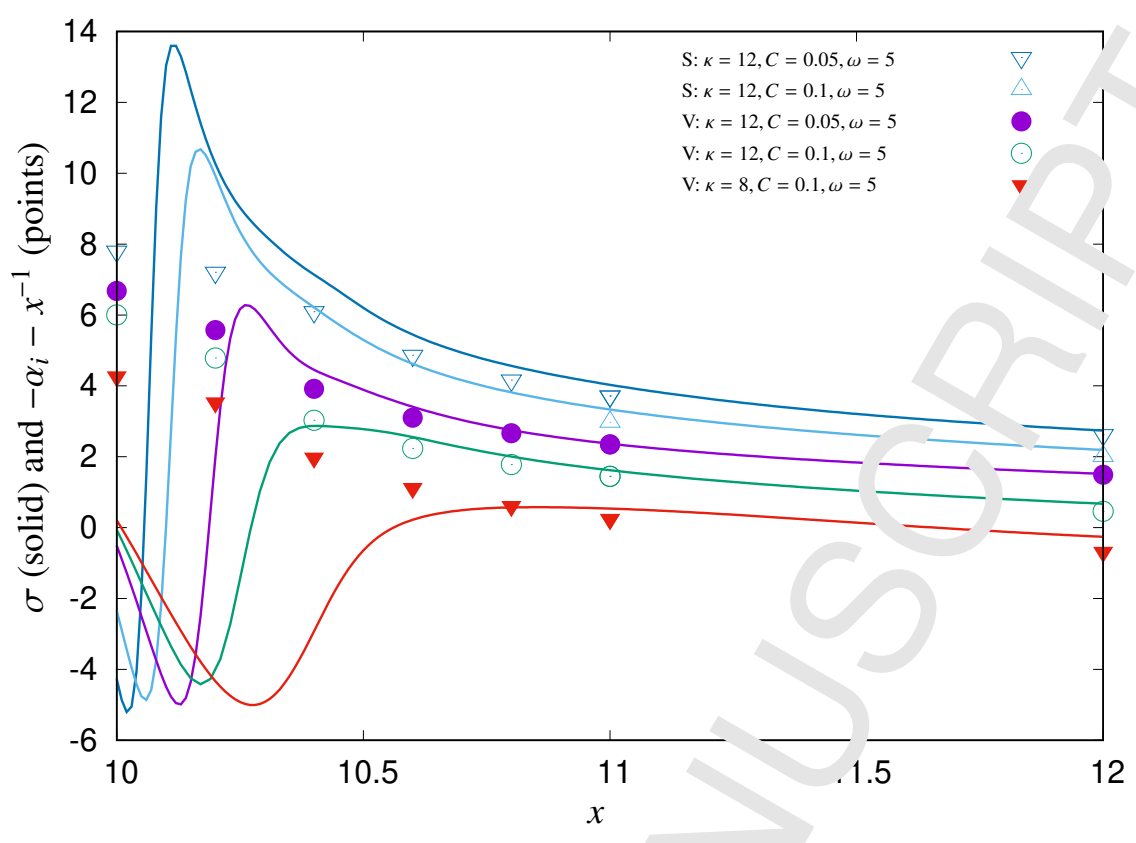

Figure 3: Spatial growth rates (14) obtained by parabolic marchino (solid) a. the analogous local eigenvalues of (15) (points) for a range of disturbances and base flows, imposed at $x=x_{0}={ }^{1} \mathrm{U} . \ldots$ key, $\mathrm{V}$ denotes varicose modes, whilst $\mathrm{S}$ denotes sinuous modes.

determined for $C=0.1$ and a wider injection mogion of $C=0.05$ (both with $\kappa=12, x_{0}=10$ ). It is clear that the streak induced by the wider injection. -egion $(C=0.05)$ is substantially more unstable with growth evident even at $x=20$, whilst the narrower injection region $(C=0.1)$ leads to a peak growth near $x=14$. The linear response has been nor talist. 'such that $|u|=1$ at $x=x_{0}$, and the perturbation is seen to be localised away from the boundary, sentred around the displaced shear layer of the streak. Figure 2 overlays the base flow speed $U$, vir contou. of $U=0.25,0.5$ and 0.75 , with the disturbance mostly centred between the 0.5 and $0.75 \mathrm{co}^{\prime}$ tou', , bo moving to higher values of $U$ as the downstream location $x$ is increased.

In figure 3 we vary both the am $/ \mathrm{r}$ de of injection $\kappa$ and the width of the injection region $c$, then determine a measure of local growth rate $\sigma \omega$ be

$$
r=\frac{1}{3} \frac{\mathcal{E}}{\mathrm{d} x} \quad \text { where } \quad \mathcal{E}=\left(\int_{\zeta=0}^{\zeta=\infty} \int_{\eta=0}^{\eta=\infty} u u^{*} \mathrm{~d} \eta \mathrm{d} \zeta\right)^{\frac{1}{2}},
$$

where the asterisk in ${ }^{\text {N: }}$ cates - complex conjugation. The downstream behaviour of $\sigma$ is shown from $x=x_{0}=10$ to $x=12$. Fc $\kappa=12$ the response is (locally) unstable $\sigma>0$ and becomes increasingly unstable as $C$ decre. ses (a widening injection region). For the values of $C$ covered, we find that the growth of unst? se sinuous modes is greater than any unstable varicose modes. For the lower injection amplitude $\kappa=$ : the str ak starts to stabilise to varicose modes. Restabilisation to sinuous modes occurs at lower values $c_{\text {: }}$;

Figure 4(a) hov s we effect of variations in the excitation frequency $\omega$. As we shall show later, at high frequency (or $r$ downstream) the growth rate scales linearly with frequency, which leads to the collapse of the shown data for $\omega=5,10$. Figure 4(b) similarly shows the influence of the excitation point moving further downstream (with values of $x_{0}=5,10,20$ ) demonstrating no qualitative change. 

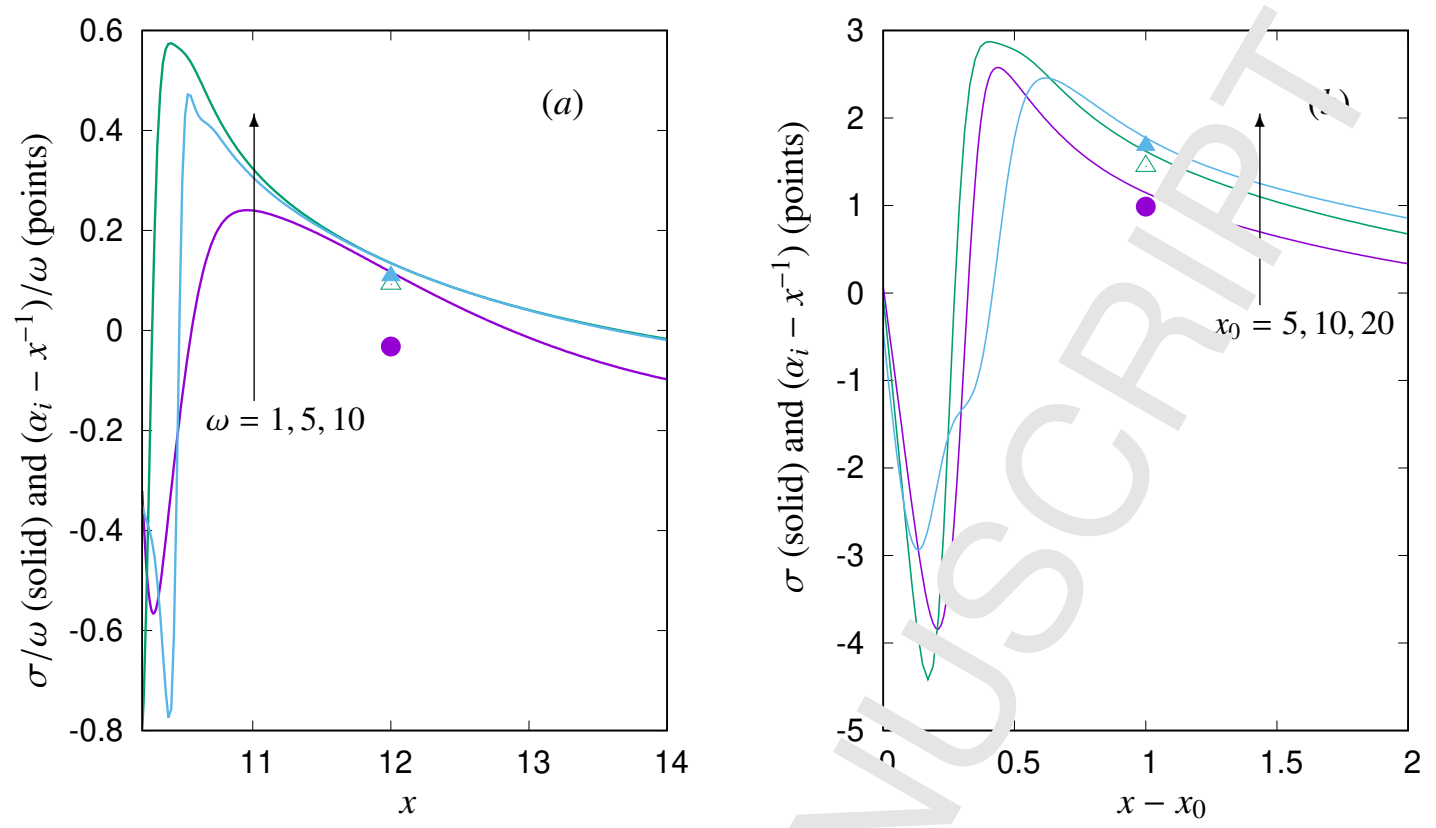

Figure 4: (a) the local (varicose) growth rate (divided by $\omega$ ) as defined by ( ${ }^{\circ}$ ) for a range of excitation frequency $\omega=1,5,10$ with $\kappa=12, C=0.1, x_{0}=10$ in (9). The growth rate scales linea ' $\mathrm{v} w, \ldots$ at high frequency. (b) the growth rate obtained for an excitation frequency of $\omega=5$, when $\kappa=12, C=0.1$ and $x_{0}=-0,20$ in (9). In both (a,b) the data points show the local weakly nonlinear eigenvalue prediction of the growth rate pre $\therefore$ by slution of the problem posed by (15).

\subsection{Local stability analysis}

On taking a weakly non-parallel approach tn the disturbance equations (11) we can seek propagating wave solutions in the form

$$
(u, \phi, \psi, \theta)=\left(x^{-} \tilde{f}(\eta, \zeta, \quad, \tilde{\phi}(\eta, \zeta ; x), \tilde{\psi}(\eta, \zeta ; x), \tilde{\theta}(\eta, \zeta ; x)) E,\right.
$$

where

$$
F=\exp \left\{i\left(\int^{x} \alpha(\xi) \mathrm{d} \xi\right)\right\}
$$

At each downstream positi ' $\mathrm{t}^{\prime}$. 1 s approach results in a bi-global eigenvalue problem for $\alpha(x)$, but of course (whilst often effec ve) th. is not fully justified asymptotically, apart from in the large- $x$ or large$\omega$ limit (as discussed be ow)

To determine the spectrum $\neg r /$ we apply the same non-uniformly distributed numerical mesh (as used for the base flow), vith a ${ }^{\circ}$ cond-order finite-difference scheme in $\zeta, \eta$. This generates a (generalised) sparse matrix eigen ’ lue prr Jlem for $(\tilde{f}, \tilde{\phi}, \tilde{\psi}, \tilde{\theta})$ and $\alpha$, associated to a given streak base flow $(U, \Phi, \Psi, \Theta)$ as defined by $\left(8^{\prime}\right.$ The tuatu1x eigenvalue problem is tackled iteratively via a shift and invert method using the SLEPc libr ry [28], to find the most dangerous spatial mode (the most negative value of $\alpha_{i}$ ).

In figure 3 we cu. ${ }^{n}$ - the local prediction of the growth rate from the parabolic marching, as provided by (14) wi ' un 2 anvalue prediction for $\alpha$. As seen from (15) there is a $x^{-1}$ attenuation of the exponential growt. $\mathrm{n} u$ and this is accounted for by comparing $\sigma$ with $-\alpha_{i}-x^{-1}$. The disturbance is centred about $x=x_{0}=1$ ) and by $x=11$ there is good quantitative agreement between the eigenvalue prediction and the parabolic marching results for $\omega=5$.

Similar comparisons can be made for variations in the excitation frequency and excitation position, as shown by the data points in figure 4 . As expected the agreement (at fixed $x$ ) is poorer at lower frequencies 

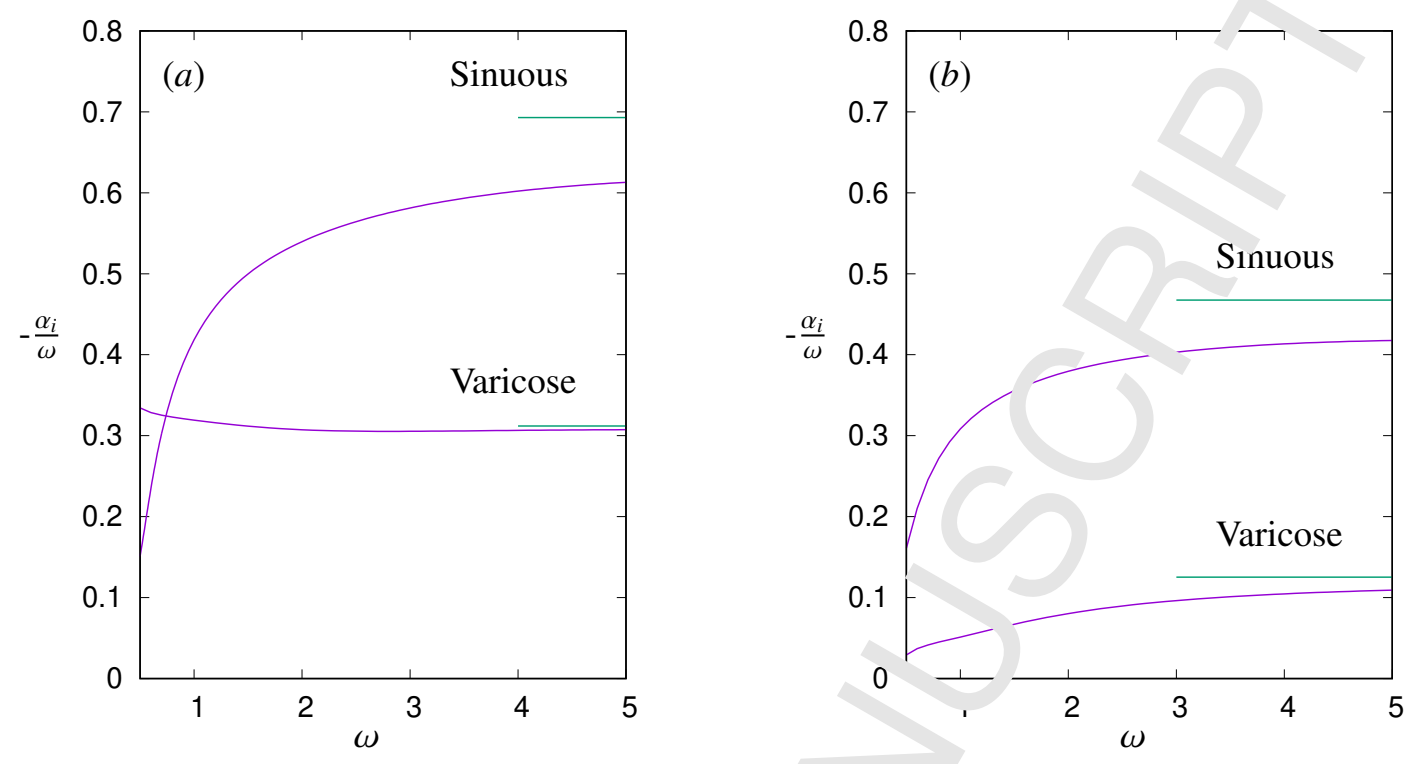

Figure 5: Spatial growth rate predictions using the local bi-global eigenvalu. problem defined by (15) with (a) $x=11$ and (b) $x=12$. In each case the asymptotic (long-wave Rayleigh) $x \gg$, rea .... of (17) is shown as a straight line segment. Both figures are for a streak induced by (9) using $\kappa=12, x_{0}=10$ and $C='$ '.

(figure 4a), although at fixed values of $\omega$ we nart ine eigenvalues to provide improved quantitative predictions as $x$ is increased. This can be observec hy rescaling $x$ in (15) in favour of a new coordinate downstream coordinate $\omega x$.

Figure 5 shows the (scaled) spatial grow 11 rate 0 tained from the eigenvalue problem for a range of frequencies at $x=11$, for the streak shown 11 Gour 1(a). Both the varicose and sinuous modes are shown, with the sinuous being most unstab 1 in this viscous problem. Further downstream at $x=12$ figure 5 (b) shows a reduction in the grow $: r$ ie fr : both classes of disturbance symmetry, with a continued dominance of the sinuous mode.

The eigenfunctions associated /ith , 5) are shown at $x=11,12$ in figures $6(\mathrm{a}-\mathrm{b})$ (varicose) and 7(a-b) (sinuous) at $\omega=5$. At thes $r$ 'wnstream positions, the eigenmodes are essentially indistinguishable from the distribution of $u$ or ainf $\downarrow$ by parabolic marching of (11), hence these are not shown.

\subsection{Large- $x$ inviscid (lr ng-1 ave) stability}

Sufficiently far downstreanı, ar neglecting inverse powers of $x$ in (11) we directly recover

$$
\begin{gathered}
2 i \alpha \tilde{f}=\tilde{\phi}_{\eta}+\tilde{\psi}_{\zeta}, \\
\tilde{\theta}=\tilde{\psi}_{\eta}-\tilde{\phi}_{\zeta}, \\
\bar{U}_{\eta} \tilde{\phi}+\bar{U}_{\zeta} \tilde{\psi}=2 i \alpha(\bar{U}-\omega / \alpha) \tilde{f}, \\
\bar{U}_{\eta} \tilde{\psi}-\bar{U}_{\zeta} \tilde{\phi}=-(\bar{U}-\omega / \alpha) \tilde{\theta},
\end{gathered}
$$

where $\bar{U}=U_{L}+\tilde{U}$ is the downstream velocity distribution of the streak. In terms of a disturbance-wave pressure

$$
p=\tilde{p}(\eta, \zeta ; x) E+\text { c.c. },
$$


the leading-order for large $x$ satisfies

$$
\begin{aligned}
& \tilde{p}_{\eta}=i \alpha(\bar{U}-\omega / \alpha) \tilde{\phi}, \\
& \tilde{p}_{\zeta}=i \alpha(\bar{U}-\omega / \alpha) \tilde{\psi} .
\end{aligned}
$$

From (16) we therefore recover the two-dimensional (long-wave limit of the) press, - - Rayleigh equation

$$
\nabla^{2} \tilde{p}=\frac{2}{\bar{U}-\omega / \alpha}\left(\tilde{p}_{\eta} \bar{U}_{\eta}+\tilde{p}_{\zeta} \bar{U}_{\zeta}\right)
$$

subject to

$$
p \rightarrow 0 \quad \text { as } \quad \eta^{2}+\zeta^{2} \rightarrow \infty
$$

as obtained in Hall \& Horseman [29], Timoshin \& Smith [30] and Hor In $_{\varepsilon}$ [31] for example, on setting a zero wavenumber.

The eigenvalue problem (17) is tackled using the same iterative span matrix formulation to determine the quantity $\omega / \alpha$, from which we can infer a value for $\alpha_{i} / \omega$. Th value rovides the asymptotic behaviour for (15) for increasing frequency or downstream position. $\mathrm{H}_{\mathrm{l}}$ ' $\mathrm{re} \supset \mathrm{s}$ shows the predictions of (17) as the horizontal line segments, and these are approached as $\omega$ innmm.

\section{4. 'Upper-branch' modes}

The previous eigenvalue problems rely on downstr $9 \mathrm{~m}$ w. velengths of the perturbation being sufficiently long compared to the local boundary-layer thicknes. - is assumption requires that $\omega \ll R e^{1 / 2} /(2 x)^{1 / 2}$. At higher frequencies $\omega$ or (equivalently) whe rffica ntly far downstream $(x \gg 1)$, the wavelength of the disturbance must eventually become compara $\sim$ to the local boundary-layer thickness. In this limit we can formally recover an eigenvalue problem for the spatial growth rate via a parallel flow assumption applied in the usual manner, by inclusior of the corresponding shorter length scale $(X)$ and faster time scale $(T)$ :

$$
X=R e^{1 / 2} x, \quad T=R e^{1 / 2} t .
$$

The harmonic behaviour of the $v$ 'ves is thın

$$
\exp \{i(\tilde{\alpha} X-\tilde{\omega} T)\},
$$

where $\tilde{\alpha}=(2 x)^{-1 / 2} \bar{\alpha}, \tilde{\omega}=(2 x,-12 \bar{\omega}$. The corresponding form of (17) is

$$
\left(\jmath^{2}-\bar{\alpha}^{2}\right) \tilde{p}=\frac{2}{\bar{U}-\bar{\omega} / \bar{\alpha}}\left(\tilde{p}_{\eta} \bar{U}_{\eta}+\tilde{p}_{\zeta} \bar{U}_{\zeta}\right),
$$

again subject to (1\&. This i the full Rayleigh pressure equation, see for example [29].

For fixed $\bar{\omega}(21)$ repı. . . s a challenging bi-global polynomial eigenvalue problem for the complex wavenumber $\bar{a}$ Rathe than dealing with this polynomial nature, we take an alternative approach. We solve (21) as a, inline $r$ problem for the unknowns of $\tilde{p}$ (at all nodal positions in the $\zeta-\eta$ plane) plus the complex vi ${ }^{\cdots} \cdot$ o of $\alpha$ via Newton iteration. To add $\bar{\alpha}$ as a single additional degree of freedom, we compensate by si $\mathrm{ti} \cdot \mathrm{g}$ one of the pressure degrees of freedom to unity; thereby normalising the eigenomde. The advantage $f$ this approach is that we avoid the polynomial nature of the eigenvalue problem, but this being an iterative method, we require a good initial guess and only recover one mode rather than a subset of the full spectrum. For the initial guess we use a long-wave eigenmode obtained from (17) at a small value of $\bar{\omega}$. 

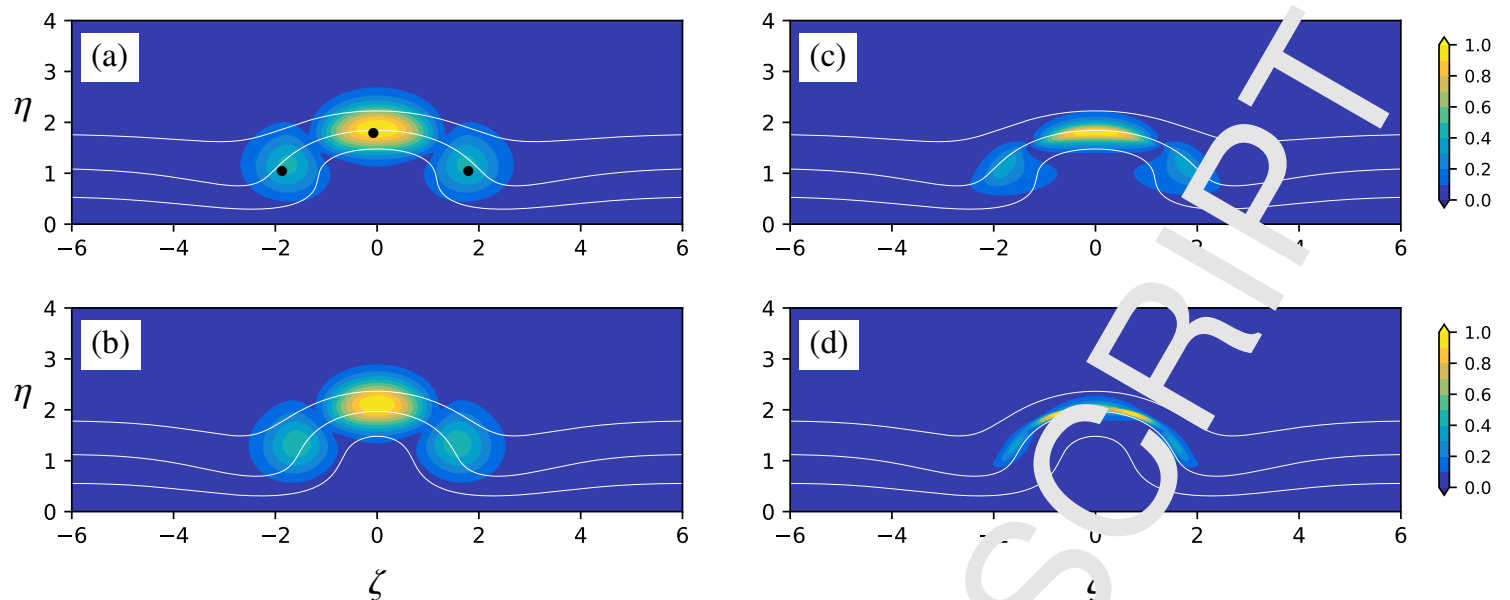

Figure 6: Varicose eigenmodes showing the absolute value of the perturbation tu he do .... stream flow velocity $(|u|)$, as obtained from the local eigenvalue problem (15) with $\omega=5$ at (a) $x=11$ and (b) $x=12$. F. 'so shown are the corresponding inviscid eigenmodes with $\bar{\omega}=0.45$ at (c) $x=11$ and (d) $x=12$. The eigenmodes - $\mathrm{e}$ each ormalised to a unit maximum, and each

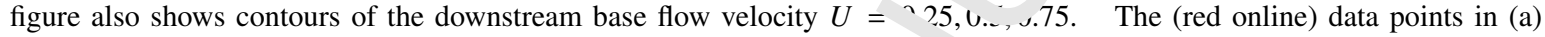
indicate maxima of experimentally obtained varicose eigenmodes at the samc 'nwnstream position, as given in figure 7(a) of [7].

Figure 8 shows the dependence of the most dangerous nc e e's growth rate $-\alpha_{i}$, as determined from (21) with $\kappa=12\left(x_{0}=10, C=0.1\right)$ over a range $\mathrm{c}$, at $=11$ and $x=12$ for both the varicose and sinuous mode. We see behaviour that is in line with $\mathrm{L}$ e c. Jerimental observations of Asai et al. [7], with a varicose instability providing a peak growth $\cdots$ - nit. $11 y$, but that the varicose mode rapidly becomes more stable as the streak develops/decays downstru. $\eta$. Further downstream the most unstable mode is sinuous, and this sinuous mode's growth rats is less sensitive to downstream position. We also see that the sinuous mode restabilises at a lower $f$ equen $'$ than the varicose mode.

Examples of the inviscid eigenmode for $u a_{2}-s h r w$ in figure 6(c,d) for varicose modes and figure 7(c,d) for sinuous modes. As in the time-m rch $\mathrm{d}$ stability results of [13], and the experimental measurements of [7] the varicose mode peak near ı. naxi num of transverse shear but there is no such simple correlation of the sinuous mode with he peak if spanwise shear. Both varicose and sinuous modes extend around the majority of the shea' lay . that is the streak boundary. A further comparison with experimental observations follows.

\section{Experimental compr isons}

The presence of comparav. orr wth rates for both varicose and sinuous instabilities near to the source of a localised streak $\mathrm{h} s$ beer observed experimentally by Asai et al. [7] and it is worthwhile to consider how our current for ulatior applies to that configuration.

The experiment vi Asaı et al. [7] placed a $7.5 \mathrm{~mm}$ wide mesh screen $500 \mathrm{~mm}$ from the leading edge of a thin plate, in 1 unifor 1 freestream flow of $4 \mathrm{~m} / \mathrm{s}$. The height of the screen was $2.4 \mathrm{~mm}$, which was also the displacemen. thic ${ }^{2}$.ess of the corresponding (undisturbed) Blasius boundary layer at $x^{*}=500 \mathrm{~mm}$. As a result a nonlinear perturbation caused by the mesh screen to the otherwise two-dimensional (Blasius) flow in isolated streak develops downstream. This streak has an aspect ratio of $O(1)$ in the cross sectional, lane, matching the scalings of (1). The stability of the streak is then examined by localised harmonic (acoustic) excitation at $x^{*} \approx 510-515 \mathrm{~mm}$.

Although no systematic study was undertaken in [9], quantitative agreement between the [7] experimental data for a steady streak has been obtained along the centreline $\left(z^{*}=0\right)$ over a range of $x^{*}=550$ to 

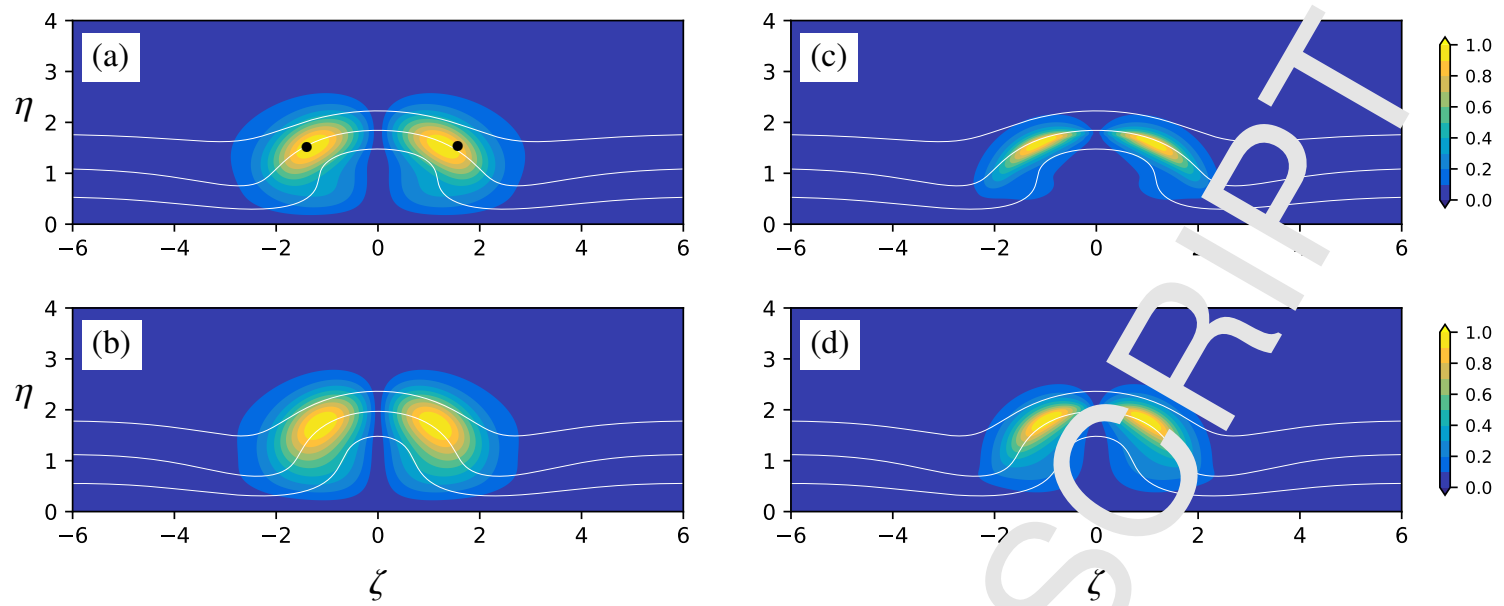

Figure 7: Sinuous eigenmodes showing the absolute value of the perturbation tu he do .... stream flow velocity $(|u|)$, as obtained from the local eigenvalue problem (15) with $\omega=5$ at (a) $x=11$ and (b) $x=12$. F. 'so shown are the corresponding inviscid eigenmodes with $\bar{\omega}=0.45$ at (c) $x=11$ and (d) $x=12$. The eigenmodes -e each ormalised to a unit maximum, and each

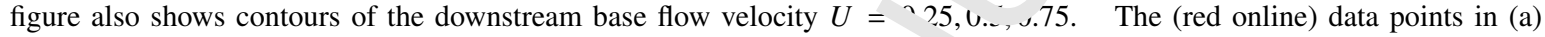
indicate maxima of experimentally obtained sinuous eigenmodes at the samt 'vwnstream position, as given in figure 7(b) of [7].

$700 \mathrm{~mm}$ with parameter values of $\kappa=12, \gamma=10, C=1 / .0$ and $x_{0}=10$ in (9). We therefore take this streak base flow as an approximation of the expe ' menta' configuration [7] and determine its (spatial) stability properties. A similar approach was taken in 'he .NS work of [13], by choosing a suitable body force term to represent the experimentally obti . $\ldots$ - $+\mathrm{r}_{\mathrm{r}} \mathrm{k}$ flow data.

For $x_{0}=10$, to relate the dimensionless problem 'erein to the experimental configuration requires $L^{*}=50 \mathrm{~mm}$, leading to a global Reynold $\ldots$. mber of $R e=13,542$. Given this Reynolds number, the dimensional cross sectional coordinates re

$$
\left(y^{*}, z^{*}\right) \cdot L^{*} \cdot e^{-1,}(2 x)^{1 / 2}(\eta, \zeta) \approx 2(\eta, \zeta) \mathrm{mm},
$$

at $x^{*}=550 \mathrm{~mm}(x=11)$. Apa $\mathrm{t}$ froı a dimensional factor of $2 \mathrm{~mm}$, the cross sectional results for $C=0.1$ and $\kappa=12$ in figures $1(, \infty, 6$ and 7 can be directly compared with the experimental data of [7].

The contours of the eigenmod component $|u|$ for this streak base flow are shown in figure 6(a,c) (varicose) and figure $7(\mathrm{a}-\mathrm{c})$ (sinv us) it $x=11$. The eigenmode shape is relatively insensitive to frequency over a broad range and (for a ralitative comparison) we also show the dimensionless position of the maxima of $|u|$ for the ex erirenta data of [7] as obtained at ( $\left.x^{*}=550 \mathrm{~mm}, x=11\right)$ with $110 \mathrm{~Hz}$ (varicose) and $60 \mathrm{~Hz}$ (sinuou. ' fr 'icin', .

A more sensitive cor purison - n be made of the spatial growth rates observed at $x^{*}=550 \mathrm{~mm}$ in figures 11 and 13 of [7] to :ither th viscous prediction of figure 5 or the inviscid results of figure 8 . The data of [7], for the baseflc of $f$ gure 1(a), are shown in figure 8 .

The lowest exc cation , equency in the experimental results is $40 \mathrm{~Hz}$. This yields a value of $\omega=\pi$ in (15), and $\omega(2 x / R e)^{1} ? \approx 0.127$ suggesting we are potentially in the viscous response regime. The relevant viscous prediction is given by figure 5 , which gives a growth rate of $0.019 \mathrm{~mm}^{-1}$ for the varicose mode. The measurc ${ }^{1} \mathrm{e}$. perimental value is $0.022 \mathrm{~mm}^{-1}$.

The peak varic e growth rate in the experimental configuration is at $110 \mathrm{~Hz}$, with $\omega(2 x / R e)^{1 / 2} \approx 0.35$. This is in the inviscid regime of figure 8 with a predicted growth rate of $0.062 \mathrm{~mm}^{-1}$ compared to the observed value of $0.055 \mathrm{~mm}^{-1}$. The peak predicted value for an inviscid varicose mode in figure 8 corresponds to a dimensional frequency of nearer $140 \mathrm{~Hz}$, with a value of $0.067 \mathrm{~mm}^{-1}$. Similarly, the sinuous 

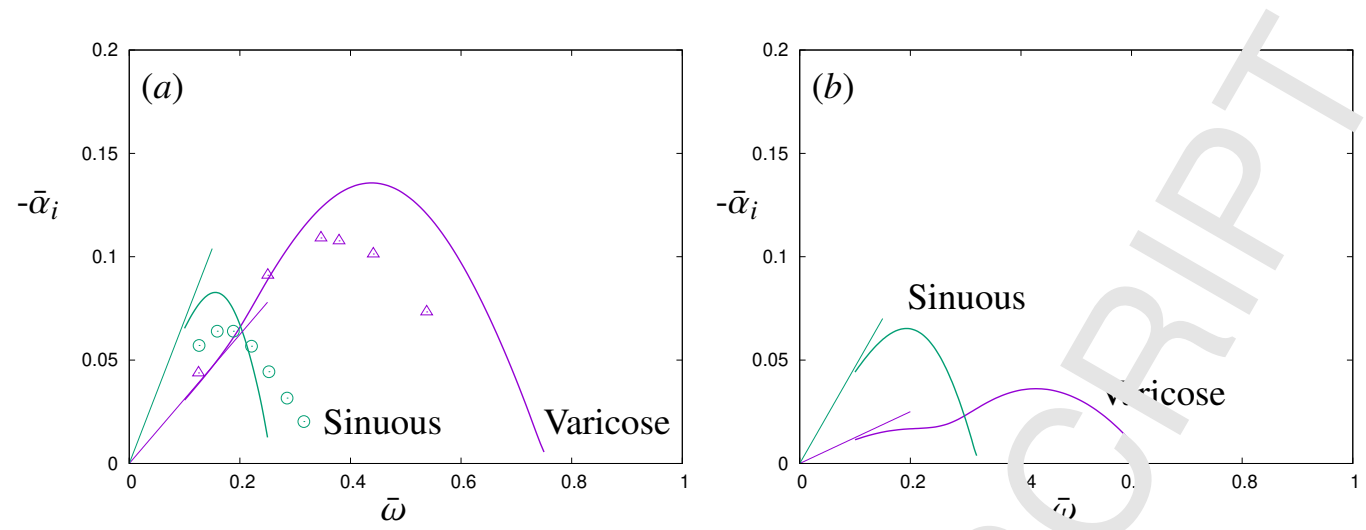

Figure 8: Spatial growth rates obtained from solution of the inviscid equation ?1 for a streak baseflow driven by $\kappa=12$, $C=0.1$ and $x_{0}=10$ in (9) evaluated at (a) $x=11$ and (b) $x=12$. In each ca the lo $\cdots$ ave $\bar{\omega} \ll 1$ asymptote is shown as a straight line segment. Data points in (a) are the observed (non-dimensionalised) $\mathrm{g}_{1}$-th rates presented in [7], at $x^{*}=550 \mathrm{~mm}$, as discussed in section 4 .

modes at $x^{*}=550 \mathrm{~mm}$ as reported by [7] give a peak groxith mon ? approximately $0.032 \mathrm{~mm}^{-1}$ at $50 \mathrm{~Hz}$. The prediction of figure 8 translates to a peak dimensiona o "

\section{Discussion}

Isolated steady low-speed streaks in a Blasius hounc ry layer can be captured by a parabolised (and asymptotically-rigorous) form of the Navier-Su'-as equations obtained in the high Reynolds number limit (the 'boundary-region equations'). Being parabolic in $x$, this governing system is an efficient method of obtaining fully nonlinear, nor para. ${ }^{\prime}$ l streaks in an otherwise two-dimensional developing layer, and are in essence the Görtler vor 'x equal ons applied to a localised structure with no curvature. Whilst there is some similarity in the s jvern $\ldots_{-}$, ystem with that of the Parabolised Navier-Stokes Equations [32], there are also some imp' stan differences. Although the latter have proved to be a popular tool, especially to the aerospace $\mathrm{cr} \mathrm{mn}_{\mathrm{n}}$. nity , being considerably faster computationally than DNS computations), their basic formulati - is not asymptotically rigorous, and this is reflected in the need for ad-hoc restrictions in the choict of nu. arical parameters.

We focus attention on streal $s$ th $t$ are driven by localised injection (but with zero net mass flux), and hence the streak decays dow ct eam, with the far-downstream flow returning to a Blasius profile. The (steady) downstream evo' $\lrcorner$ tion ol . streak has been addressed previously in [9]. This work shows that the same governing equatir as $\mathrm{i}$ the unsteady form (4) can also be used to formulate parabolic linearised perturbation equations $\left(11, v^{\circ}\right.$.h can be computed in tandem with the (streak) base flow. Downstream of a (temporally) $\mathrm{p}$ ' riodic 'isturbance generator, we obtain 'transient' growth of perturbations prior to their ultimate decay as the treak amplitude itself decreases. We also formulate a weakly non-parallel eigenvalue appr $\ldots$ h to ur spatial stability (15) of the streak, again based on the same boundary-region formulation ar 1 demo, strate consistency with the spatially marched evolution of a perturbation sufficiently far from he dir urbance generator.

As the freq enc $f$ any (linear) disturbance generator increases, or equivalently if a disturbance is followed sufficic ly far downstream, we pass through a long-wavelength Rayleigh region (17), and yet further downstre.$m$, we ultimately recover the full pressure Rayleigh equation for the disturbance (21).

For the streaks examined herein, the sinuous modes are always more dangerous for sufficiently long downstream wavelengths. However, as the frequency of excitation increases, in the Rayleigh regime, we find that varicose modes can dominate the response near the injection region centred at $x=x_{0}$. 
However, the peak growth rate of the inviscid varicose mode decays more rapidly dowr ,tream and again the sinuous mode eventually dominates; this is clearly seen in figure 8 .

We conclude that isolated streak flows are well captured by the system (4) and al' on for effective predictions of both the steady streak and its spatial instability. The parabolic nature $f$ ( 4 in the associated $R e \gg 1$ limit allows for an efficient computation of the streak/stability flow fea res. 's spite the ad-hoc modelling of the experimental streak generation mechanism as a localised injertion, $\therefore$ quantitative predictions have a good level of consistency with observations. In addition $\mathrm{w}^{f}$ alsr .. nver the qualitative features of (i) dominance of a varicose mode near $x=x_{0}$, (ii) more rapid dec." sf the varicose instability for increasing $x-x_{0}$ and a changeover to sinuous modes as the most ur caole anc (iii) restabilisation of the sinuous mode at a lower frequency than the varicose mode.

\section{References}

[1] J.-C. Loiseau, J.-C. Robinet, S. Cherubini, E. Leriche, Invesı_-atiun of the roughness-induced transition: global stability analyses and direct numerical simulations J. Fluid Mech. 760 (2014) 175211.

[2] M. A. Bucci, D. K. Puckert, C. Andriano, J.-C. T - s..., . Cherubini, J.-C. Robinet, U. Rist, Roughness-induced transition by quasi-resonance of a varicose global mode, J. Fluid Mech. 836 (2018) 167-191.

[3] V. Citro, F. Giannetti, P. Luchini, F. Auteri, 'oba tability and sensitivity analysis of boundarylayer flows past a hemispherical roughness ele. 'elı, Phys. Fluids 27 (8) (2015) 084110.

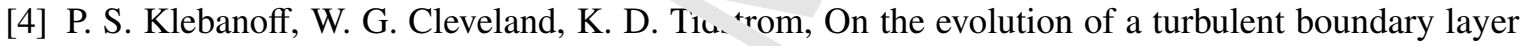
induced by a three-dimensional roughness element, J. Fluid Mech. 237 (1992) 101-187.

[5] N. Gregory, W. S. Walker, The effe t of tral sition of isolated surface excrescences in the boundary layer, Tech. Rep. R. \& M. 2779.

[6] N. De Tullio, P. Paredes, N. C Sa dha 1 , V. Theofilis, Laminar-turbulent transition induced by a discrete roughness element $\mathrm{j}$. $\mathrm{a} \mathrm{su}_{\mathrm{r}}{ }^{\mathrm{r}}$, snic boundary layer, J. Fluid Mech. 735 (2013) 613-646.

[7] M. Asai, M. Minagawa, M. Nı' 'ioka, The instability and breakdown of a near-wall low-speed streak, J. Fluid Mech. 4r , ?002) 289-314.

[8] R. D. Joslin, C. E. Gros ' Growth characteristics downstream of a shallow bump: Computation and experiment, $\mathrm{Pr} / \mathrm{s}$. F'uids 7 (12) (1995) 3042-3047.

[9] R. E. Hewitt, P. W. L . -k Localised streak solutions for a Blasius boundary layer, J. Fluid Mech. 849 (2018) $88-901$.

[10] C. Cossu, L. Bra. dt $r_{n}$ Tollmien-Schlichting-like waves in streaky boundary layers, Eur. J. Mech.B/Fluids` 3 (6) (`004) 815-833.

[11] J. H. M. F. necr . L, L. Brandt, A. Talamelli, C. Cossu, Experimental study of the stabilization of

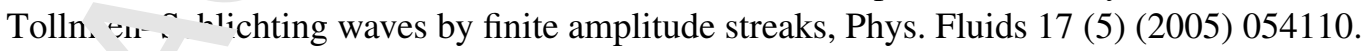

[12] L. L. Van Tommelen, R. Yapalparvi, Laminar boundary-layer separation control by Görtler-scale blowing, Eur. J. Mech.-B/Fluids 46 (2014) 1-16.

[13] L. Brandt, Numerical studies of the instability and breakdown of a boundary-layer low-speed streak, Eur. J. Mech.-B/Fluids 26 (1) (2007) 64-82. 
[14] E. Piot, G. Casalis, U. Rist, Stability of the laminar boundary layer flow enco atering a row of roughness elements: biglobal stability approach and DNS, Eur. J. Mech.-B/F' 1 ids .7 (6) (2008) 684-706.

[15] S. Cherubini, M. D. De Tullio, P. De Palma, G. Pascazio, Transient growth $1 \omega^{\text {th }}$, flow past a threedimensional smooth roughness element, J. Fluid Mech. 724 (2013) 642-6 ?

[16] R. E. Hewitt, P. W. Duck, Three-dimensional boundary layers with sh ,rt s ‥ wise scales, J. Fluid Mech. 756 (2014) 452-469.

[17] S. N. Brown, K. Stewartson, On similarity solutions of the bounda $y$-laye equations with algebraic decay, J. Fluid Mech. 23 (04) (1965) 673-687.

[18] R. E. Hewitt, P. W. Duck, S. R. Stow, Continua of states in 'oun 'v-layer flows, J. Fluid Mech. 468 (2002) 121-152.

[19] A. J. Williams, R. E. Hewitt, Micro-slot injection into a bou. rary layer driven by a favourable pressure gradient, J. Eng. Math. 107 (2017) 19-35.

[20] R. E. Hewitt, P. W. Duck, A. J. Williams, Injection intu houndary layers: solutions beyond the classical form, J. Fluid Mech. 822 (2017) 617-639.

[21] M. Higuera, J. M. Vega, Modal description of : . . n nol notimal streaks, J. Fluid Mech. 626 (2009) $21-31$.

[22] P. Hall, The nonlinear development of Görtler ' $\mathrm{O}_{1}$ : ${ }^{\text {res in }}$ growing boundary layers, J. Fluid Mech. 193 (1988) 243-266.

[23] P. Luchini, Reynolds-number-independent insta sility of the boundary layer over a flat surface, J. Fluid Mech. 327 (1996) 101-116.

[24] P. Luchini, Reynolds-number-indep - dent i stability of the boundary layer over a flat surface: optimal perturbations, J. Fluid Mer n. 404 ( 1 , (2000) 289-309.

[25] P. Andersson, M. Berggren. L. ' $\mathrm{H}^{\prime}$ nningson, Optimal disturbances and bypass transition in boundary layers, Phys. Flui s 11 (1) (1999) 134-150.

[26] P. Hall, S. Sherwin, Streamwise vutices in shear flows: harbingers of transition and the skeleton of coherent structures, J. ' tuid Mech. 661 (2010) 178-205.

[27] P. R. Amestoy, I. S. \uff, J. V. L'Excellent, Multifrontal parallel distributed symmetric and unsymmetric solvers, Co ıp. ' lethods in Appl. Mech. and Eng. 184 (2) (2000) 501-520.

[28] V. Hernandez, I G. Ku - in, V. Vidal, SLEPc: A scalable and flexible toolkit for the solution of eigenvalue prr olems, . CM Trans. Math. Software 31 (3) (2005) 351-362.

[29] P. Hall, N. I Hor .....n, The linear inviscid secondary instability of longitudinal vortex structures in bound; cy laye. , J. Fluid Mech. 232 (1991) 357-375.

[30] S. N. Timu.:- F. T. Smith, Singular modes in Rayleigh instability of three-dimensional strean. v1sr . . tex flows, J. Fluid Mech. 333 (1997) 139-160.

[31] L. M. Ho 'ing, Long wavelength disturbances to non-planar parallel flow, J. Fluid Mech. 31 (4) (1968) 625-634.

[32] T. Herbert, Parabolized stability equations, Ann. Rev. Fluid Mech. 29 (1) (1997) 245-283. doi:10.1146/annurev.fluid.29.1.245. 